\title{
A realistic study of 3D composition of carbon nanotubes and carbonaceous nanocompounds from different soils around coal power plant
}

\author{
Marcos L.S. Oliveira, Omar Ramirez, Ismael L. Schneider, Elba C. Teixeira, Luis F.O. Silva
}

\begin{abstract}
Information on the effects of multi-walled carbon nanotubes (MWCNTs) on topsoil around coal power plants (CPPs) is still very limited. In the present work, the influences of MWCNTs on potential hazardous elements (PHEs) and environmental carbonaceous compounds in agrarian topsoil around CPPs of Latin America were investigated. The environmentally elevated proportions of MWCNTs and PHEs can cause damage to developing a fetus. The ecological impacts of industrial energy byproducts generated by MWCNTs were also studied. The surface morphologies of MWCNTs and PHEs detected in topsoil samples were analyzed by advanced electron microscopy in a combination of energy dispersive X-ray spectroscopy (EDS). The alterations could be originated due to the different geophysical constituents and superficial structure, which in turn disturbed their geoavailability in studied topsoil. It was found that a large amount of MWCNTs and amorphous carbonaceous matters, which are responsible for adsorbing PHEs, existed in soils around CPPs. Hence, these findings could be used to better understand the geochemical properties of PHEs near CPPs.
\end{abstract}

Keywords:

Potential hazardous elements, Agrarian topsoil, Natural carbonaceous matter, Terrestrial environment, Coal impacts, Ecological danger 